\title{
Physical Characteristics of Stream Subbasins \\ in the Middle Minnesota-Little Cottonwood River Basin, South-Central Minnesota
}

By Christopher A. Sanocki

\begin{abstract}
Data that describe the physical characteristics of stream subbasins upstream from selected sites on streams in the Middle Minnesota-Little Cottonwood River Basin, located in south-central Minnesota are presented in this report. The physical characteristics are the drainage area of the subbasin, the percentage area of the subbasin covered only by lakes, the percentage area of the subbasin covered by both lakes and wetlands, the main-channel length, and the mainchannel slope. Stream sites include outlets of subbasins of at least 5 square miles, outfalls of sewage treatment plants, and locations of U.S. Geological Survey low-flow, high-flow, and continuous-record gaging stations.
\end{abstract}

\section{Introduction}

This is the 11th report in a series detailing subbasin characteristics of streams in Minnesota and adjacent states. The Middle Minnesota-Little Cottonwood River Basin drains an area of 1,350 square miles and is represented by hydrologic accounting unit 07020007 (U.S. Geological Survey, 1974). The Middle MinnesotaLittle Cottonwood River Basin includes parts of Cottonwood, Redwood, Renville, Brown, Sibley, Blue Earth, Nicollet, and Le Sueur Counties in south-central Minnesota.

Selected data for sites on streams at outlets of subbasins larger than about 5 square miles; at outfalls of sewage treatment plants; and at locations of U.S. Geological Survey (USGS) low-flow, high-flow, and continuous-record gaging stations located in the Middle Minnesota-Little Cottonwood River Basin are presented in this report.

This report was prepared in cooperation with the Minnesota Department of Transportation.

\section{Acknowledgments}

Thomas E. Kujawa, a graduate student at Mankato State University, did much of the digitizing and assisted in the preparation of this report. The Water Resource Center at Mankato State University provided detailed watershed boundaries, which were used for parts of this report. These contributions were essential for the completion of this report.

\section{Methods}

U.S. Geological Survey 7-1/2 minute series topographic maps were used as source maps to obtain the areas for the subbasin boundaries, lakes, marshes, the main-channel length, and the contour elevation points used in this report. Paper copies of the maps were used. A geographic information system (GIS) was used to define the geographic location and extent of the subbasins, lakes, marshes, main-channels, and elevation points. Data digitized from paper copies were in error by no more than twice the horizontal accuracy of National Mapping Standards of 40 feet (Thompson, 1987, p. 104). All thematic (digitized) data were projected into an Albers Equal-Area projection for storage and analysis.

Subbasin boundaries were delineated on the basis of anthropogenic activities and topographic contours. Anthropogenic activities, such as the installation of storm sewers, the drainage of wetlands, and the diversion of streams, may alter the drainage area of a stream. Data from fieid inspections and recent drainageditch maps, therefore, were transferred to the topographic maps. The subbasin boundaries were digitized by the Minnesota State Planning Land Management Information Center, Mankato State University, and the U.S. Geological Survey Minnesota using a GIS.

Lake and marsh data were digitized using a GIS. Lake and marsh boundaries were overlaid on the subbasin boundaries to associate each lake and marsh 
with a subbasin. The total area of lakes and marshes within each subbasin was calculated by the GIS. Total marsh area plus total lake area is defined as storage area. Lakes and marshes were digitized by the U.S. Geological Survey Minnesota.

Main channels were delineated for each subbasin on the 7-1/2 minute topographic maps starting at the outflow of the subbasin and continuing upstream. Whenever the main channel joined with another stream, the stream upstream of the junction that drained the largest area was selected as the main channel. The main channel, which represents the watercourse that drains the greatest area, is continuous and is defined as a single trace that passes through marshes, lakes, and midline of rivers and braided streams from the basin outlet to an endpoint in the basin, generally at the basin divide. The main channels were digitized by the Minnesota Department of Transportation, using a CAD system and transferred to the GIS. Stream extensions which represent a portion of the main channel from the end of the mapped stream (blue line on USGS $71 / 2$ minute maps) to an endpoint within the basin, generally at the basin divide, were digitized by U.S. Geological Survey Minnesota using a GIS. The main-channel data were overlaid onto the subbasin data to associate each main channel with its subbasin.

Elevation points were digitized at the intersection of topographic contour lines and main channels. The elevation data were digitized using a GIS. The elevation data was overlaid onto the main channel data to associate each elevation data point with a main channel. Two points on the main channel, at 10 percent and at 85 percent of the main channel length from the basin outlet to the drainage divide, were located by the GIS. The elevations of these two points were interpolated from the digitized elevation data. Main-channel slope was calculated by dividing the difference in elevation between these points by the distance along the stream channel between these points.

\section{Physical Characteristics of Middle Minnesota-Little Cottonwood River Subbasins}

Physical characteristics determined for each of the subbasins shown on plate 1 are presented in table 1 . Subbasins are presented in order from headwaters to mouth. The rank of the subbasin stream is shown by indentation; whenever two subbasin streams joined, the stream draining the least cumulative area was assigned a lower rank and indented in the table.
The data for drainage area, and main-channel length, are reported using three significant figures or rounded to the nearest one-hundredth of a unit. The data for lake area and storage area are reported using two significant figures or rounded to the nearest one-tenth of a percent. The data for main-channel slope is reported to the nearest one-tenth of a foot.

The following is an explanation of terms used in table 1:

Subbasin number. A seven digit number based on the Minnesota Common Stream and Watershed Numbering System (Minnesota Department of Natural Resources, 1981). The first two digits are 28 and identify the Middle Minnesota-Little Cottonwood River Basin. The following five digits are arbitrary and are used to identify each individual subbasin.

Stream name. The name of the stream or ditch shown on U.S. Geological Survey 7-1/2 minute topographic maps. The relative position of the subbasin above other subbasins, streams, gaging stations, and outfalls from sewage treatment plants also is given.

Outlet location. The U.S. Public Lands Survey System is used to describe the location where the stream exits the subbasin, down to quarter-quarter section. The description includes quarter-quarter section, section, township, and range.

Drainage area. That area, measured on a horizontal plane, enclosed by a topographic divide, within which direct surface runoff from precipitation normally flows by gravity into a watercourse above a specific point. This may include closed basins and other areas that do not contribute directly to surface runoff.

Lake area. The percentage of the drainage area covered by open water as shown on 7-1/2 minute topographic maps.

Storage area. The percentage of a drainage area covered by open water and marshes as shown on 7-1/2 minute topographic maps. Marsh areas are not shown on plate 1.

Main-channel length. The total length of the main channel from the basin outlet to a point within the basin (generally at the basin divide) representing the watercourse that drains the greatest area.

Main-channel slope. The average slope of the watercourse between the points at 10 and at 85 percent of the distance along the main channel from the basin outlet to the drainage divide.

Stream extension. A representation of the main channel from the end of the mapped stream line (blue line on USGS $71 / 2$ minute series maps) to an endpoint 
within the basin, generally at the basin divide. This is done by interpreting topographic relief so that the extension of the main channel represents the water course draining the greatest area.

\section{References Cited}

Minnesota Department of Natural Resources, 1981, The Common Stream And Watershed Numbering System: Minnesota Department of Natural Resources Stream Inventory and Data Retrieval Systems Report 7002, unpaged.

Thompson, M.M., 1987, Maps for America, 3d edition: U.S. Geological Survey, 265 p.

U.S. Geological Survey, 1974, Hydrologic unit map-1974 State of Minnesota: 1 plate, scale 1:500,000. 
Table 1. Physical characteristics for the Middle Minnesota - Little Cottonwood River Basin.

[All cities and towns are in Minnesota; --, not determined]

\begin{tabular}{|c|c|c|c|c|c|c|c|c|c|c|c|c|c|}
\hline \multirow[b]{2}{*}{$\begin{array}{c}\text { Basin } \\
\text { number }\end{array}$} & \multirow[b]{2}{*}{ Stream name and location } & \multicolumn{4}{|c|}{ Outlet location } & \multicolumn{3}{|c|}{ By subbasin } & \multicolumn{5}{|c|}{ Cumulative to mouth of basin } \\
\hline & & $\begin{array}{l}\text { Quarter- } \\
\text { quarter } \\
\text { section }\end{array}$ & Section & $\begin{array}{l}\text { Town- } \\
\text { ship }\end{array}$ & Range & $\begin{array}{l}\text { Drainage } \\
\text { area } \\
\text { (square } \\
\text { miles) }\end{array}$ & $\begin{array}{c}\text { Lake } \\
\text { area } \\
\text { (percent } \\
\text { of } \\
\text { subbasin } \\
\text { area) }\end{array}$ & $\begin{array}{l}\text { Storage } \\
\text { area } \\
\text { (percent } \\
\text { of } \\
\text { subbasin } \\
\text { area) }\end{array}$ & $\begin{array}{l}\text { Drainage } \\
\text { area } \\
\text { (square } \\
\text { miles) }\end{array}$ & $\begin{array}{c}\text { Lake } \\
\text { area } \\
\text { (percent } \\
\text { of } \\
\text { subbasin } \\
\text { area) }\end{array}$ & $\begin{array}{l}\text { Storage } \\
\text { area } \\
\text { (percent } \\
\text { of } \\
\text { subbasin } \\
\text { area) }\end{array}$ & $\begin{array}{l}\text { Main } \\
\text { channel } \\
\text { length } \\
\text { (miles) }\end{array}$ & $\begin{array}{c}\text { Main } \\
\text { channel } \\
\text { slope } \\
\text { (foot } \\
\text { per mile) }\end{array}$ \\
\hline & 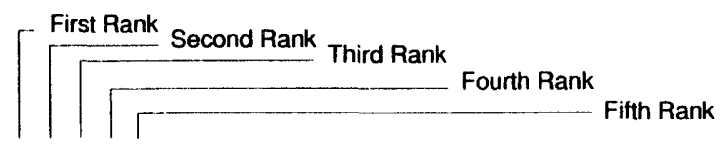 & & & & & & & & & & & & \\
\hline 2506000 & Minnesota River above Beaver Creek & $\mathrm{NE}^{1 / 4} \mathrm{SW}^{1 / 4}$ & 27 & $113 \mathrm{~N}$ & $35 \mathrm{~W}$ & 8,930 . & 3.3 & 6.5 & 8,930 & 3.3 & 6.5 & 226. & 1.8 \\
\hline 2801100 & Minnesota River above Crow Creek & $\mathrm{NE}^{1 / 4} \mathrm{SE}^{1 / 4}$ & 35 & $113 \mathrm{~N}$ & $35 \mathrm{~W}$ & 9.59 & .9 & 3.7 & 8,940 . & 3.2 & 6.5 & 230. & 1.8 \\
\hline 2809900 & County Ditch No. 22 to Crow Creek above mouth & $\mathrm{NE}^{1 / 4} \mathrm{SE}^{1 / 4}$ & 04 & $112 \mathrm{~N}$ & $35 \mathrm{~W}$ & 16.6 & 0 & 0 & 16.6 & 0 & 0 & 11.1 & 5.4 \\
\hline 2809800 & $\begin{array}{l}\text { Crow Creek (County Ditch No. 52) to Minnesota } \\
\text { River above mouth }\end{array}$ & $\mathrm{NE}^{1 / 4} \mathrm{SE}^{1 / 4}$ & 35 & $113 N$ & $35 \mathrm{~W}$ & 20.0 & 0 & .2 & 36.6 & 0 & .1 & 15.2 & 14.2 \\
\hline 2801201 & $\begin{array}{l}\text { Minnesota River above outfall from sewage treatment } \\
\text { plant for Morton }\end{array}$ & $\mathrm{NW}^{1 / 4} \mathrm{SW}^{1 / 4}$ & 31 & $113 N$ & $34 \mathrm{~W}$ & 2.14 & 1.1 & 3.2 & 8,980 & 3.2 & 6.5 & 233. & 1.8 \\
\hline 2801200 & Minnesota River above Birch Coulee Creek & $\mathrm{NW}^{1} / 4 \mathrm{SW}^{1} / 4$ & 05 & $112 \mathrm{~N}$ & $34 \mathrm{~W}$ & 4.67 & .5 & 1.0 & $8,980$. & 3.2 & 6.5 & 236. & 1.8 \\
\hline 2800100 & County Ditch No. 124 above County Ditch No. 85A & $\mathrm{NE}^{1 / 4} \mathrm{SE}_{4}^{1 / 4}$ & 32 & $114 \mathrm{~N}$ & $34 \mathrm{~W}$ & 17.6 & 0 & 0 & 17.6 & 0 & 0 & 8.94 & 7.5 \\
\hline 2800200 & $\begin{array}{l}\text { County Ditch No. 85A to County Ditch No. } 124 \\
\text { above mouth }\end{array}$ & $\mathrm{NE}^{1} / 4 \mathrm{SE}^{1 / 4}$ & 32 & $114 N$ & $34 \mathrm{~W}$ & 10.7 & 0 & 0 & 10.7 & 0 & 0 & 9.49 & 7.1 \\
\hline 2800300 & Judicial Ditch No. 12 above subbasin 2801300 & $\mathrm{SW}^{1} / 4 \mathrm{SE}^{1 / 4}$ & 01 & $.113 N$ & $34 \mathrm{~W}$ & 10.8 & 0 & 0 & 10.8 & 0 & 0 & 7.41 & 7.9 \\
\hline 2801300 & $\begin{array}{l}\text { Judicial Ditch No. } 12 \text { to Birch Coulee Creek above } \\
\text { mouth }\end{array}$ & $\mathrm{NE}^{1} / 4 \mathrm{NE}^{1 / 4}$ & 29 & $113 N$ & $34 \mathrm{~W}$ & 15.6 & 0 & .2 & 26.4 & 0 & .1 & 14.9 & 9.2 \\
\hline 2801001 & $\begin{array}{l}\text { Birch Coulee Creek (Judicial Ditch No. 124) above } \\
\text { gaging station near Morton: station number is } \\
05316590\end{array}$ & $\mathrm{SW}^{1 / 4} \mathrm{SW}^{1 / 4}$ & 33 & $113 \mathrm{~N}$ & $34 \mathrm{~W}$ & 13.0 & 0 & 0 & 67.7 & 0 & .1 & 21.2 & 11.0 \\
\hline 2801000 & Birch Coulee Creek to Minnesota River above mouth & $\mathrm{NW}^{1} / \mathrm{SW}^{1} / 4$ & 05 & $112 \mathrm{~N}$ & $34 \mathrm{~W}$ & 0.16 & 0 & 0 & 67.8 & 0 & .1 & 22.1 & 12.7 \\
\hline 2801600 & $\begin{array}{l}\text { County Ditch No. 109A to Purgatory Creek above } \\
\text { mouth }\end{array}$ & $\mathrm{SW}^{1 / 1} \mathrm{SW}^{1 / 4}$ & 02 & $112 \mathrm{~N}$ & $34 \mathrm{~W}$ & 12.2 & 0 & 0 & 12.2 & 0 & 0 & 7.56 & 10.6 \\
\hline 2801400 & Purgatory Creek to Minnesota River above mouth & $\mathrm{NW}^{1} / 4 \mathrm{SW}^{1 / 4}$ & 11 & $112 \mathrm{~N}$ & $34 \mathrm{~W}$ & 8.08 & 0 & .2 & 20.3 & 0 & .1 & 9.88 & 29.3 \\
\hline 2802701 & $\begin{array}{l}\text { Minnesota River above outfall from sewage treatment } \\
\text { plant for Franklin }\end{array}$ & $\mathrm{NW}^{1} / 4 \mathrm{SE}^{1 / 4}$ & 11 & $112 \mathrm{~N}$ & $34 \mathrm{~W}$ & 5.24 & .2 & 2.8 & 9,070 . & 3.2 & 6.4 & 242 . & 1.7 \\
\hline
\end{tabular}


Table 1. Physical characteristics for the Middle Minnesota - Little Cottonwood River Basin-Continued.

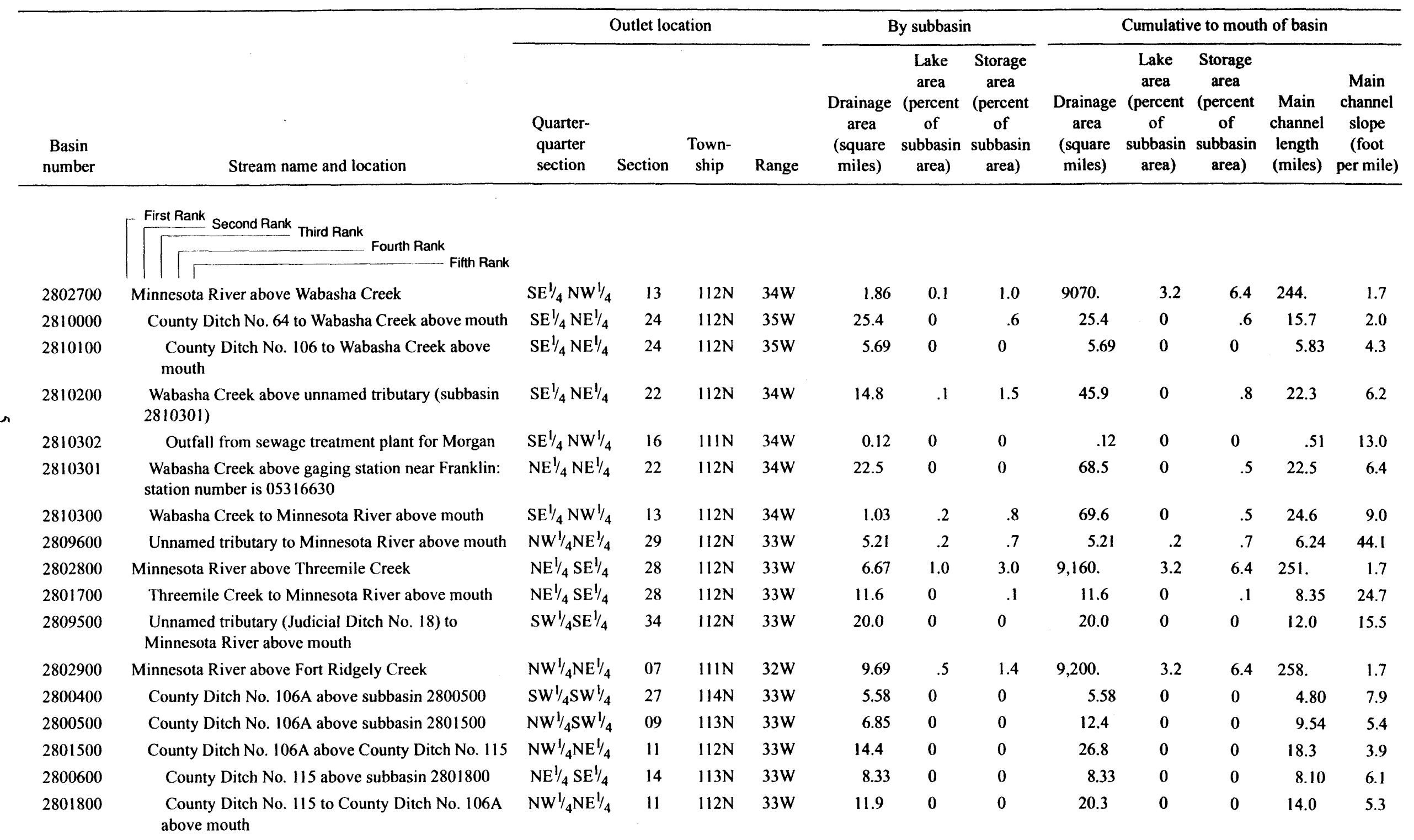


Table 1. Physical characteristics for the Middle Minnesota - Little Cottonwood River Basin-Continued.

\begin{tabular}{|c|c|c|c|c|c|c|c|c|c|c|c|c|c|}
\hline \multirow[b]{2}{*}{$\begin{array}{c}\text { Basin } \\
\text { number }\end{array}$} & \multirow[b]{2}{*}{ Stream name and location } & \multicolumn{4}{|c|}{ Outlet location } & \multicolumn{3}{|c|}{ By subbasin } & \multicolumn{5}{|c|}{ Cumulative to mouth of basin } \\
\hline & & $\begin{array}{l}\text { Quarter- } \\
\text { quarter } \\
\text { section }\end{array}$ & Section & $\begin{array}{l}\text { Town- } \\
\text { ship }\end{array}$ & Range & $\begin{array}{c}\text { Drainage } \\
\text { area } \\
\text { (square } \\
\text { miles) }\end{array}$ & $\begin{array}{c}\text { Lake } \\
\text { area } \\
\text { (percent } \\
\text { of } \\
\text { subbasin } \\
\text { area) }\end{array}$ & $\begin{array}{l}\text { Storage } \\
\text { area } \\
\text { (percent } \\
\text { of } \\
\text { subbasin } \\
\text { area) }\end{array}$ & $\begin{array}{l}\text { Drainage } \\
\text { area } \\
\text { (square } \\
\text { miles) }\end{array}$ & $\begin{array}{c}\text { Lake } \\
\text { area } \\
\text { (percent } \\
\text { of } \\
\text { subbasin } \\
\text { area) }\end{array}$ & $\begin{array}{l}\text { Storage } \\
\text { area } \\
\text { (percent } \\
\text { of } \\
\text { subbasin } \\
\text { area) }\end{array}$ & $\begin{array}{c}\text { Main } \\
\text { channel } \\
\text { length } \\
\text { (miles) }\end{array}$ & $\begin{array}{c}\text { Main } \\
\text { channel } \\
\text { slope } \\
\text { (foot } \\
\text { per mile) }\end{array}$ \\
\hline & First Rank Second Rank Third Rank & & & & & & & & & & & & \\
\hline 2801900 & $\begin{array}{l}\text { Fort Ridgely Creek (County Ditch No. 106A) above } \\
\text { County Ditch No. } 3\end{array}$ & $\mathrm{NE}^{1} / 4 \mathrm{NE}^{1 / 4}$ & 24 & $112 \mathrm{~N}$ & $33 W$ & 7.66 & 0.0 & 0.1 & 54.8 & 0.0 & 0.0 & 21.6 & 4.3 \\
\hline 2802101 & $\begin{array}{l}\text { County Ditch No. } 3 \text { above outfall from sewage } \\
\text { treatment plant for Fairfax }\end{array}$ & $\mathrm{SE}^{1} / 4 \mathrm{NE}^{1} / 4$ & 17 & $112 \mathrm{~N}$ & $32 \mathrm{~W}$ & .43 & 0 & 0 & .43 & 0 & 0 & 1.28 & 14.7 \\
\hline 2802100 & $\begin{array}{l}\text { County Ditch No. } 3 \text { to Fort Ridgely Creek above } \\
\text { mouth }\end{array}$ & $\mathrm{NE}^{1 / 4} \mathrm{NE}^{1 / 4}$ & 24 & $112 \mathrm{~N}$ & $33 \mathrm{~W}$ & 3.45 & 0 & 3.0 & 3.89 & $\mathbf{0}$ & 2.6 & 4.64 & 12.3 \\
\hline 2802201 & $\begin{array}{l}\text { Fort Ridgely Creek above gaging station near } \\
\text { Fairfax: station number is } 05316680\end{array}$ & $\mathrm{NW}^{1 / 4} \mathrm{SW}^{1 / 4}$ & 05 & $111 \mathrm{~N}$ & $32 \mathrm{~W}$ & 10.4 & 0 & .8 & 69.0 & 0 & .3 & 27.4 & 7.6 \\
\hline 2802200 & Fort Ridgely Creek to Minnesota River above mouth & $\mathrm{NW}_{4}^{1} \mathrm{NE}_{4}^{1 / 4}$ & 07 & $111 \mathrm{~N}$ & $32 \mathrm{~W}$ & .23 & 0 & 0 & 69.3 & 0 & .3 & 28.3 & 8.8 \\
\hline 2803000 & Minnesota River above Spring Creek & $\mathrm{NW}^{1} / 4 \mathrm{SE}^{1 / 4}$ & 21 & $111 \mathrm{~N}$ & $32 \mathrm{~W}$ & 9.54 & 0 & .1 & 9,280 . & 3.1 & 6.3 & 266. & 1.7 \\
\hline 2809402 & $\begin{array}{l}\text { County Ditch No. } 12 \text { above gaging station near } \\
\text { Evan: station number is } 05316690\end{array}$ & $\mathrm{NE}^{1 / 4} \mathrm{NW}^{1 / 4}$ & 25 & $111 \mathrm{~N}$ & $33 W$ & 4.13 & 0 & $\mathbf{0}$ & 4.13 & 0 & 0 & 3.70 & 7.7 \\
\hline 2809401 & $\begin{array}{l}\text { Spring Creek (Judicial Ditch No. 29) above gaging } \\
\text { station near Sleepy Eye: station number is } 05316700\end{array}$ & $\mathrm{NE}^{1 / 4} \mathrm{SE}^{1 / 4}$ & 24 & $111 \mathrm{~N}$ & $33 W$ & 28.7 & 0 & 0 & 32.8 & 0 & 0 & 16.5 & 2.7 \\
\hline 2809400 & Spring Creek to Minnesota River above mouth & $\mathrm{NW} / 4 \mathrm{SE}^{1 / 4}$ & 21 & $111 \mathrm{~N}$ & $32 \mathrm{~W}$ & 12.1 & .8 & .8 & 44.9 & .2 & .2 & 20.4 & 10.1 \\
\hline 2809300 & $\begin{array}{l}\text { Unnamed tributary (County Ditch No. 13) to } \\
\text { Minnesota River above mouth }\end{array}$ & $\mathrm{SE} / 4 \mathrm{NE}^{1} / 4$ & 27 & $111 \mathrm{~N}$ & $32 \mathrm{~W}$ & 11.3 & 0 & .6 & 11.3 & 0 & 6 & 8.57 & 22.5 \\
\hline 2809200 & $\begin{array}{l}\text { Unnamed tributary (County Ditch No. 10) to } \\
\text { Minnesota River above mouth }\end{array}$ & $\mathrm{NW}^{1} / 4 \mathrm{NW}^{1 / 4}$ & 31 & $111 \mathrm{~N}$ & $31 \mathrm{~W}$ & 13.3 & 0 & .2 & 13.3 & 0 & .2 & 8.36 & 26.4 \\
\hline 2807900 & Minnesota River above Little Rock Creek & $\mathrm{SW}^{1} /{ }_{4} \mathrm{SW}^{1} / 4$ & 30 & $111 \mathrm{~N}$ & $31 \mathrm{~W}$ & 8.06 & .8 & 2.9 & 9,350 & 3.1 & 6.3 & 273. & 1.7 \\
\hline 2800800 & $\begin{array}{l}\text { County Ditch No. } 27 \text { to County Ditch No. } 34 \\
\text { above mouth }\end{array}$ & $\mathrm{NE}^{1} / 4 \mathrm{NE}^{1 / 4}$ & 28 & $113 \mathrm{~N}$ & $32 \mathrm{~W}$ & 7.36 & 0 & .6 & 7.36 & 0 & .6 & 5.57 & 6.8 \\
\hline 2800700 & $\begin{array}{l}\text { County Ditch No. } 34 \text { to Judicial Ditch No. } 8 \text { above } \\
\text { mouth }\end{array}$ & $\mathrm{NW}^{1 / 1} \mathrm{NE}^{1 / 4}$ & 34 & $113 \mathrm{~N}$ & $32 \mathrm{~W}$ & 15.3 & .1 & .3 & 22.7 & 0 & .4 & 13.8 & 3.7 \\
\hline
\end{tabular}


Table 1. Physical characteristics for the Middle Minnesota - Little Cottonwood River Basin-Continued.

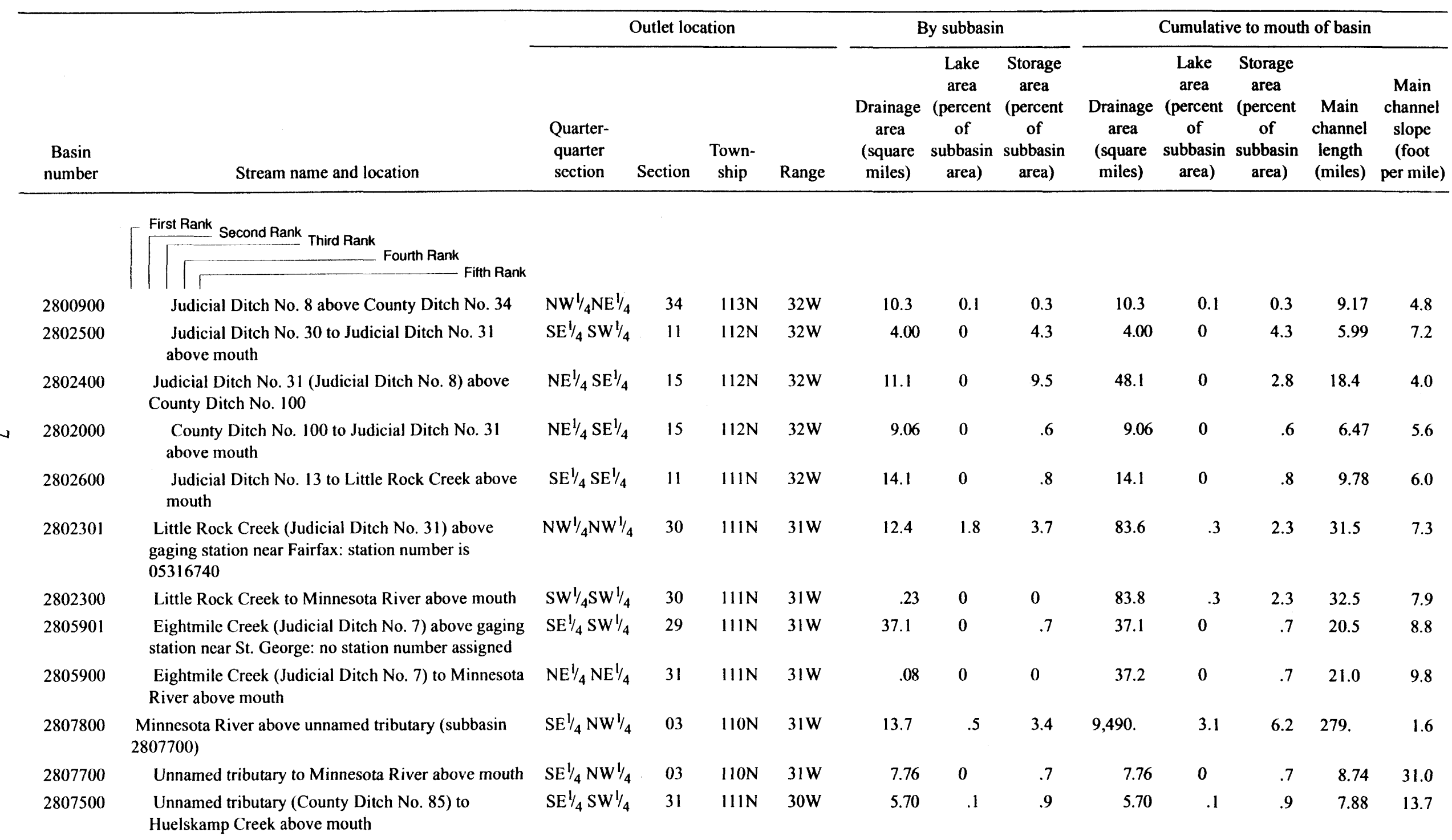


Table 1. Physical characteristics for the Middle Minnesota - Little Cottonwood River Basin—Continued.

\begin{tabular}{|c|c|c|c|c|c|c|c|c|c|c|c|c|c|}
\hline \multirow[b]{2}{*}{$\begin{array}{l}\text { Basin } \\
\text { number }\end{array}$} & \multirow[b]{2}{*}{ Stream name and location } & \multicolumn{4}{|c|}{ Outlet location } & \multicolumn{3}{|c|}{ By subbasin } & \multicolumn{5}{|c|}{ Cumulative to mouth of basin } \\
\hline & & $\begin{array}{l}\text { Quarter- } \\
\text { quarter } \\
\text { section }\end{array}$ & Section & $\begin{array}{l}\text { Town- } \\
\text { ship }\end{array}$ & Range & $\begin{array}{l}\text { Drainage } \\
\text { area } \\
\text { (square } \\
\text { miles) }\end{array}$ & $\begin{array}{c}\text { Lake } \\
\text { area } \\
\text { (percent } \\
\text { of } \\
\text { subbasin } \\
\text { area) }\end{array}$ & $\begin{array}{l}\text { Storage } \\
\text { area } \\
\text { (percent } \\
\text { of } \\
\text { subbasin } \\
\text { area) }\end{array}$ & $\begin{array}{l}\text { Drainage } \\
\text { area } \\
\text { (square } \\
\text { miles) }\end{array}$ & $\begin{array}{c}\text { Lake } \\
\text { area } \\
\text { (percent } \\
\text { of } \\
\text { subbasin } \\
\text { area) }\end{array}$ & $\begin{array}{c}\text { Storage } \\
\text { area } \\
\text { (percent } \\
\text { of } \\
\text { subbasin } \\
\text { area) }\end{array}$ & $\begin{array}{l}\text { Main } \\
\text { channel } \\
\text { length } \\
\text { (miles) }\end{array}$ & $\begin{array}{c}\text { Main } \\
\text { channel } \\
\text { slope } \\
\text { (foot } \\
\text { per mile) }\end{array}$ \\
\hline & First Rank Second Rank Third Rank & & & & & & & & & & & & \\
\hline 2807401 & $\begin{array}{l}\text { Huelskamp Creek (County Ditch No. } 80 \text { ) above } \\
\text { gaging station near Klossner: no station number } \\
\text { assigned }\end{array}$ & $\mathrm{SW}^{1} /{ }_{4} \mathrm{SE}^{1 / 4}$ & 06 & $110 \mathrm{~N}$ & $30 \mathrm{~W}$ & 5.96 & 0.0 & 0.3 & 11.7 & 0.1 & 0.6 & 9.24 & 23.6 \\
\hline 2807400 & Huelskamp Creek to Minnesota River above mouth & $\mathrm{NE}^{1} / 4 \mathrm{SE}^{1 / 4}$ & 07 & $110 \mathrm{~N}$ & $30 \mathrm{~W}$ & .40 & 0.0 & 0.0 & 12.1 & .1 & .6 & 10.1 & 26.9 \\
\hline 2807600 & Minnesota River above Fritsche Creek & $\mathrm{NW}^{1} /{ }_{4} \mathrm{NW}^{1} / 4$ & 17 & $110 \mathrm{~N}$ & $30 \mathrm{~W}$ & 14.3 & .5 & 6.9 & 9,520 & 3.1 & 6.2 & 286. & 1.6 \\
\hline 2807200 & $\begin{array}{l}\text { Fritsche Creek (County Ditch No. } 77 \text { ) to Minnesota } \\
\text { River above mouth }\end{array}$ & $\mathrm{NW}^{1} / 4 \mathrm{NW}^{1} / 4$ & 17 & $110 \mathrm{~N}$ & $30 \mathrm{~W}$ & 20.7 & 0 & 1.0 & 20.7 & 0 & 1.0 & 15.6 & 13.8 \\
\hline 2807302 & $\begin{array}{l}\text { Minnesota River above gaging station near New Ulm: } \\
\text { station number is } 05316770\end{array}$ & $\mathrm{NE}^{1 / 4} \mathrm{NE}^{1 / 4}$ & 20 & $110 \mathrm{~N}$ & $30 \mathrm{~W}$ & 3.49 & .2 & .7 & $9,550$. & 3.0 & 6.2 & 288. & 1.6 \\
\hline 2807301 & $\begin{array}{l}\text { Minnesota River above outfall from sewage treatment } \\
\text { plant for New Ulm }\end{array}$ & $\mathrm{SE}^{1} / 4 \mathrm{NW}^{1} / 4$ & 34 & $110 \mathrm{~N}$ & $30 \mathrm{~W}$ & 3.63 & .6 & 1.2 & 9,550 . & 3.0 & 6.2 & 293. & 1.5 \\
\hline 2807100 & $\begin{array}{l}\text { Heymans Creek (County Ditch No. 38) to Minnesota } \\
\text { River above mouth }\end{array}$ & $\mathrm{NW}^{1} / 4 \mathrm{SE}^{1 / 4}$ & 34 & $110 \mathrm{~N}$ & $30 \mathrm{~W}$ & 16.6 & 0 & .8 & 16.6 & 0 & .8 & 10.7 & 12.7 \\
\hline 2807300 & Minnesota River above Cottonwood River & $\mathrm{SE}^{1 / 4} \mathrm{SE}^{1 / 4}$ & 34 & $110 \mathrm{~N}$ & $30 \mathrm{~W}$ & .81 & 0 & .3 & 9,570 & 3.0 & 6.2 & 295 & 1.5 \\
\hline 2900100 & Cottonwood River above mouth & $\mathrm{SE}^{1 / 4} \mathrm{SE}^{1 / 4}$ & 34 & $110 \mathrm{~N}$ & $30 \mathrm{~W}$ & 1,310 & .6 & 1.6 & 1,310 & .6 & 1.6 & 156. & 4.7 \\
\hline 2805800 & Minnesota River above Little Cottonwood River & $\mathrm{NW} / /{ }_{4} \mathrm{NW}^{1} / 4$ & 16 & $109 \mathrm{~N}$ & $29 \mathrm{~W}$ & 17.2 & 1.2 & 3.6 & 10,900 . & 2.7 & 5.6 & 301. & 1.5 \\
\hline 2808000 & $\begin{array}{l}\text { Little Cottonwood River above unnamed tributary } \\
\text { (subbasin } 2808100 \text { ) }\end{array}$ & $\mathrm{NW}^{1 / 1} \mathrm{SE}^{1 / 4}$ & 15 & $108 \mathrm{~N}$ & $34 \mathrm{~W}$ & 34.6 & 0 & .2 & 34.6 & 0 & .2 & 32.5 & 16.1 \\
\hline 2808100 & $\begin{array}{l}\text { Unnamed tributary to Little Cottonwood River } \\
\text { above mouth }\end{array}$ & $\mathrm{NW}^{1 / 4} \mathrm{SE}^{1 / 4}$ & 15 & $108 \mathrm{~N}$ & $34 \mathrm{~W}$ & 7.82 & 0 & 3.4 & 7.82 & 0 & 3.4 & 8.72 & 15.5 \\
\hline 2809700 & $\begin{array}{l}\text { Little Cottonwood River above County Ditch No. 28- } \\
1\end{array}$ & $\mathrm{NE}^{1} / 4 \mathrm{SE}^{1} / 4$ & 17 & $108 \mathrm{~N}$ & $33 W$ & 15.5 & 0 & 1.0 & 57.9 & 0 & .9 & 37.5 & 14.6 \\
\hline 2809001 & $\begin{array}{l}\text { Unnamed tributary above outfall from sewage } \\
\text { treatment plant for Comfrey }\end{array}$ & $\mathrm{SE} / 4 \mathrm{SW} / 4$ & 35 & $108 \mathrm{~N}$ & $34 \mathrm{~W}$ & .45 & 0 & 0 & .45 & 0 & 0 & 1.82 & 15.2 \\
\hline
\end{tabular}


Table 1. Physical characteristics for the Middle Minnesota - Little Cottonwood River Basin —Continued.

\begin{tabular}{|c|c|c|c|c|c|c|c|c|c|c|c|c|c|}
\hline \multirow[b]{2}{*}{$\begin{array}{l}\text { Basin } \\
\text { number }\end{array}$} & \multirow[b]{2}{*}{ Stream name and location } & \multicolumn{4}{|c|}{ Outlet location } & \multicolumn{3}{|c|}{ By subbasin } & \multicolumn{5}{|c|}{ Cumulative to mouth of basin } \\
\hline & & $\begin{array}{l}\text { Quarter- } \\
\text { quarter } \\
\text { section }\end{array}$ & Section & $\begin{array}{l}\text { Town- } \\
\text { ship }\end{array}$ & Range & $\begin{array}{c}\text { Drainage } \\
\text { area } \\
\text { (square } \\
\text { miles) }\end{array}$ & $\begin{array}{c}\text { Lake } \\
\text { area } \\
\text { (percent } \\
\text { of } \\
\text { subbasin } \\
\text { area) }\end{array}$ & $\begin{array}{c}\text { Storage } \\
\text { area } \\
\text { (percent } \\
\text { of } \\
\text { subbasin } \\
\text { area) }\end{array}$ & $\begin{array}{c}\text { Drainage } \\
\text { area } \\
\text { (square } \\
\text { miles) }\end{array}$ & $\begin{array}{c}\text { Lake } \\
\text { area } \\
\text { (percent } \\
\text { of } \\
\text { subbasin } \\
\text { area) }\end{array}$ & $\begin{array}{c}\text { Storage } \\
\text { area } \\
\text { (percent } \\
\text { of } \\
\text { subbasin } \\
\text { area) }\end{array}$ & $\begin{array}{l}\text { Main } \\
\text { channel } \\
\text { length } \\
\text { (miles) }\end{array}$ & $\begin{array}{c}\text { Main } \\
\text { channel } \\
\text { slope } \\
\text { (foot } \\
\text { per mile) }\end{array}$ \\
\hline & rst Rank Second Rank Third Rank & & & & & & & & & & & & \\
\hline & $\Gamma_{\Gamma}$ Fourth Rank Fifth Rank & & & & & & & & & & & & \\
\hline 2809000 & $\begin{array}{l}\text { Unnamed tributary (County Ditch No. 39) to } \\
\text { County Ditch No. 28-1 above mouth }\end{array}$ & $\mathrm{SE} / 4 \mathrm{SW}^{1 / 4}$ & 17 & $108 \mathrm{~N}$ & $33 \mathrm{~W}$ & 16.6 & 0.0 & 0.4 & 17.0 & 0.0 & 0.4 & 16.5 & 10.2 \\
\hline 2809100 & $\begin{array}{l}\text { County Ditch No. 28-1 to Little Cottonwood River } \\
\text { above mouth }\end{array}$ & $\mathrm{NE} / 4 \mathrm{SE}^{1} / 4$ & 17 & $108 \mathrm{~N}$ & $33 \mathrm{~W}$ & 6.79 & 0 & .1 & 23.8 & $\mathbf{0}$ & .3 & 17.0 & 10.4 \\
\hline 2808501 & $\begin{array}{l}\text { Little Cottonwood River above gaging station near } \\
\text { Leavenworth: station number is } 05317150\end{array}$ & $\mathrm{NE}^{1 / 4} \mathrm{SE}^{1 / 4}$ & 09 & $108 \mathrm{~N}$ & $33 \mathrm{~W}$ & 4.42 & 0 & 1.1 & 86.2 & 0 & .7 & 39.8 & 13.9 \\
\hline 2808500 & $\begin{array}{l}\text { Little Cottonwood River above unnamed tributary } \\
\text { (subbasin 2808400) }\end{array}$ & $\mathrm{NW}^{1 / 4} \mathrm{NE}^{1 / 4}$ & 32 & $109 \mathrm{~N}$ & $31 \mathrm{~W}$ & 28.4 & 1.6 & 5.6 & 115. & .4 & 1.9 & 60.4 & 9.0 \\
\hline 2808400 & $\begin{array}{l}\text { Unnamed tributary to Little Cottonwood River } \\
\text { above mouth }\end{array}$ & $\mathrm{NW}^{1 / 4} \mathrm{NE}^{1 / 4}$ & 32 & $109 \mathrm{~N}$ & $31 \mathrm{~W}$ & 7.12 & 0 & 1.2 & 7.12 & 0 & 1.2 & 6.88 & 7.4 \\
\hline 2808800 & $\begin{array}{l}\text { Unnamed tributary to Little Cottonwood River } \\
\text { above mouth }\end{array}$ & $\mathrm{SE}^{1} / 4 \mathrm{NE}^{1 / 4}$ & 28 & $109 \mathrm{~N}$ & $31 \mathrm{~W}$ & 7.11 & 1.0 & 9.9 & 7.11 & 1.0 & 9.9 & 5.97 & 7.2 \\
\hline 2808600 & $\begin{array}{l}\text { County Ditch No. } 11 \text { to Little Cottonwood River } \\
\text { above mouth }\end{array}$ & $\mathrm{NE}^{1 / 4} \mathrm{NE}^{1 / 4}$ & 28 & $109 \mathrm{~N}$ & $31 \mathrm{~W}$ & 10.1 & .5 & 7.8 & 10.1 & .5 & 7.8 & 9.26 & 5.8 \\
\hline 2808700 & Little Cottonwood River above County Ditch No. 58 & $\mathrm{SW}^{1 / 4} \mathrm{SE}^{1} / 4$ & 20 & $109 \mathrm{~N}$ & $30 \mathrm{~W}$ & 10.3 & 1.3 & 3.1 & 149. & .5 & 2.8 & 71.0 & 7.7 \\
\hline 2808900 & $\begin{array}{l}\text { County Ditch No. } 58 \text { to Little Cottonwood River } \\
\text { above mouth }\end{array}$ & $\mathrm{SW}^{1 / 4} \mathrm{SE}^{1 / 4}$ & 20 & $109 N$ & $30 \mathrm{~W}$ & 7.47 & .3 & 3.0 & 7.47 & .3 & 3.0 & 6.09 & 10.0 \\
\hline 2805702 & $\begin{array}{l}\text { Little Cottonwood River above gaging station near } \\
\text { Cambria: station number is } 05317195\end{array}$ & $\mathrm{NW}^{1 / 4} \mathrm{NE}^{1 / 4}$ & 19 & $109 \mathrm{~N}$ & $29 \mathrm{~W}$ & 10.9 & .1 & 1.6 & 168. & .5 & 2.7 & 80.3 & 6.9 \\
\hline 2805701 & $\begin{array}{l}\text { Little Cottonwood River above gaging station near } \\
\text { Courtland: station number is } 05317200\end{array}$ & $\mathrm{SW}^{1 / 4} \mathrm{NE} / 4$ & 17 & $109 \mathrm{~N}$ & $29 \mathrm{~W}$ & 2.02 & .1 & .1 & 170. & .5 & 2.7 & 82.3 & 6.9 \\
\hline 2805700 & $\begin{array}{l}\text { Little Cottonwood River to Minnesota River above } \\
\text { mouth }\end{array}$ & $\mathrm{NW}^{1} / 4 \mathrm{NW}^{1 / 4}$ & 16 & $109 \mathrm{~N}$ & $29 \mathrm{~W}$ & 0.16 & 0 & 0 & 170. & .5 & 2.7 & 82.9 & 6.9 \\
\hline 2808300 & Judicial Ditch No. 10 above County Ditch No. 63 & $\mathrm{SE}^{1} / 4 \mathrm{SW}^{1} / 4$ & 01 & $108 \mathrm{~N}$ & $30 \mathrm{~W}$ & 22.5 & 1.9 & 2.3 & 22.5 & 1.9 & 2.3 & 15.5 & 2.9 \\
\hline
\end{tabular}


Table 1. Physical characteristics for the Middle Minnesota - Little Cottonwood River Basin-Continued.

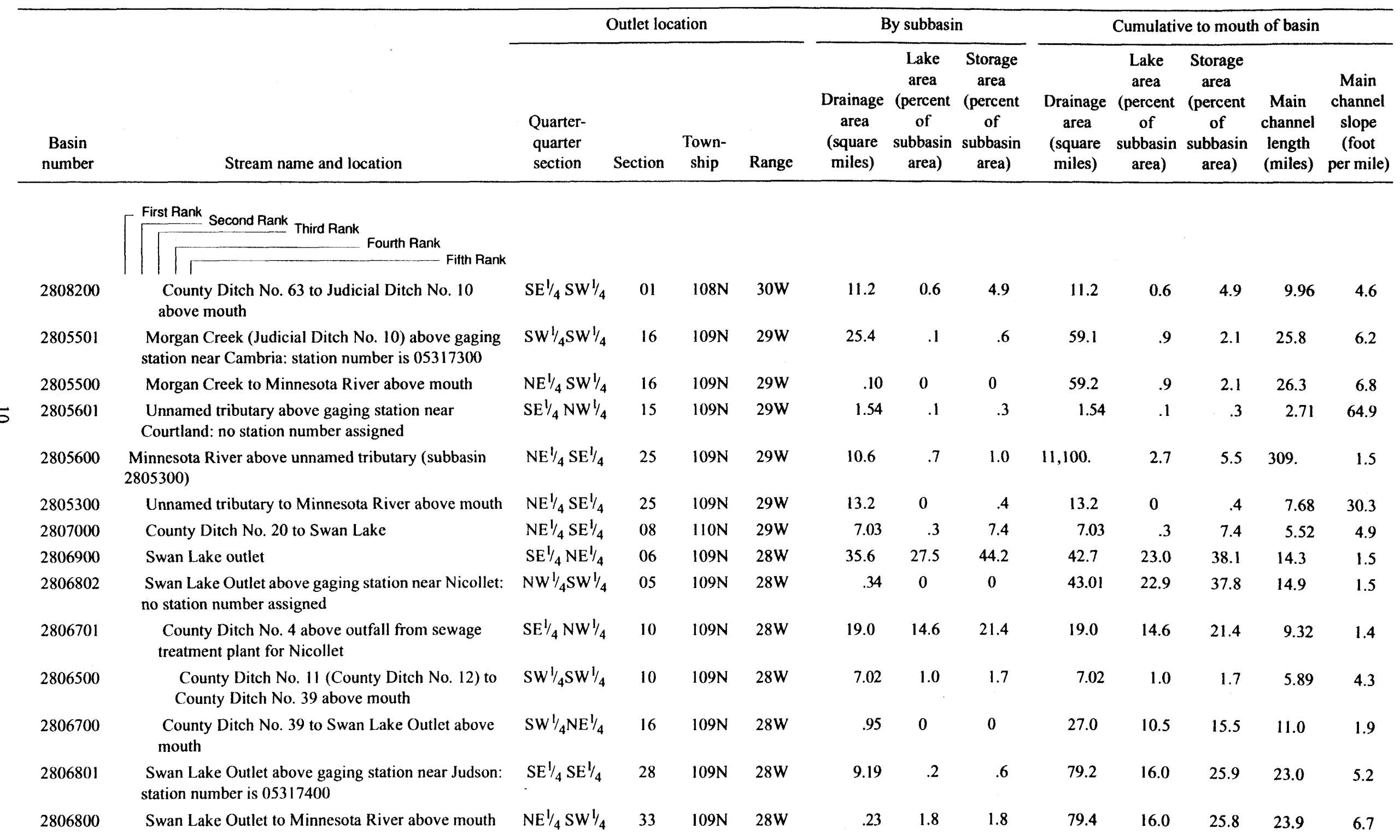


Table 1. Physical characteristics for the Middle Minnesota - Little Cottonwood River Basin-Continued.

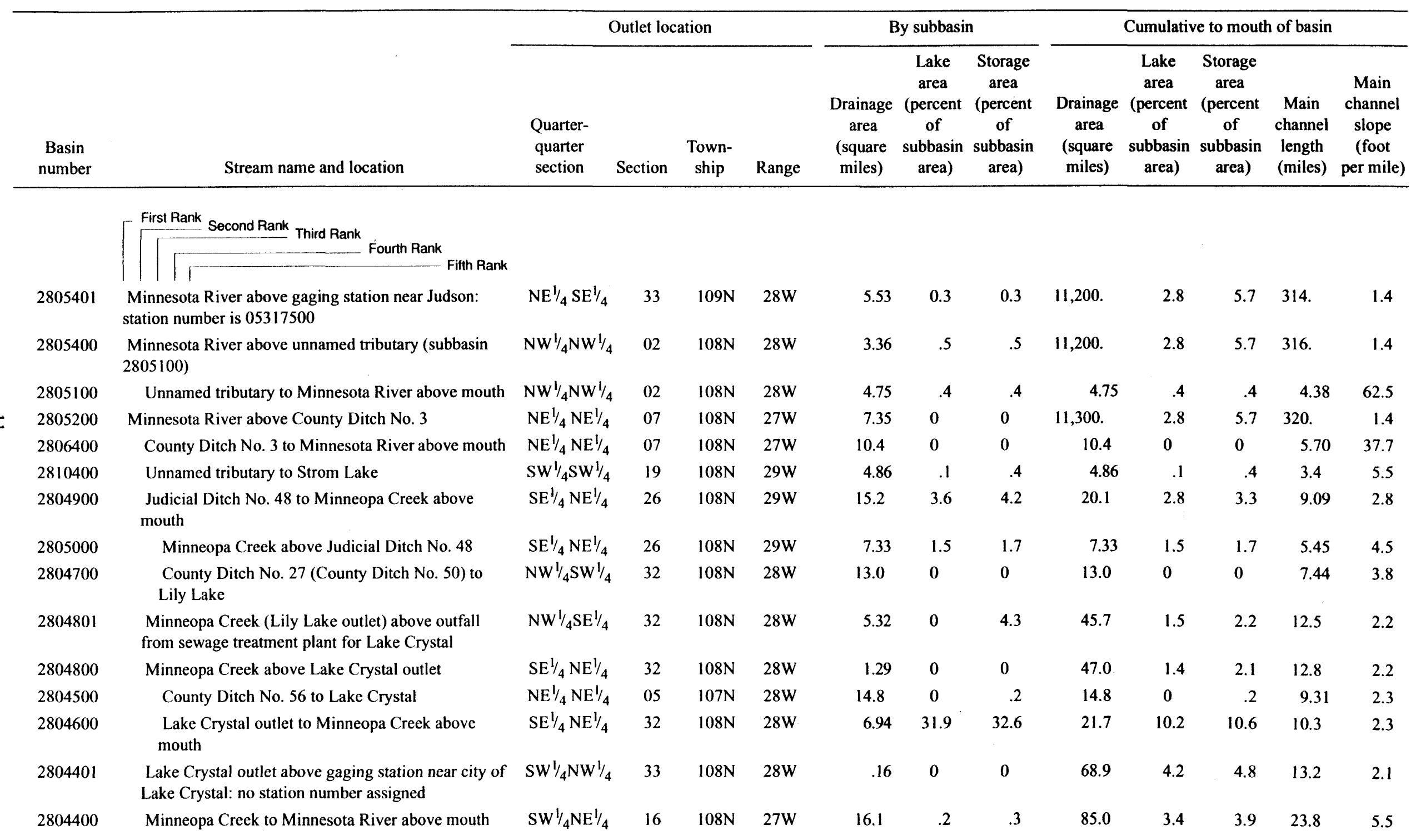


Table 1. Physical characteristics for the Middle Minnesota -Little Cottonwood River Basin-Continued.

\begin{tabular}{|c|c|c|c|c|c|c|c|c|c|c|c|c|c|}
\hline \multirow[b]{2}{*}{$\begin{array}{c}\text { Basin } \\
\text { number }\end{array}$} & \multirow[b]{2}{*}{ Stream name and location } & \multicolumn{4}{|c|}{ Outlet location } & \multicolumn{3}{|c|}{ By subbasin } & \multicolumn{5}{|c|}{ Cumulative to mouth of basin } \\
\hline & & $\begin{array}{l}\text { Quarter- } \\
\text { quarter } \\
\text { section }\end{array}$ & Section & $\begin{array}{l}\text { Town- } \\
\text { ship }\end{array}$ & Range & $\begin{array}{c}\text { Drainage } \\
\text { area } \\
\text { (square } \\
\text { miles) }\end{array}$ & $\begin{array}{c}\text { Lake } \\
\text { area } \\
\text { (percent } \\
\text { of } \\
\text { subbasin } \\
\text { area) }\end{array}$ & $\begin{array}{l}\text { Storage } \\
\text { area } \\
\text { (percent } \\
\text { of } \\
\text { subbasin } \\
\text { area) }\end{array}$ & $\begin{array}{c}\text { Drainage } \\
\text { area } \\
\text { (square } \\
\text { miles) }\end{array}$ & $\begin{array}{l}\text { Lake } \\
\text { area } \\
\text { (percent } \\
\text { of } \\
\text { subbasin } \\
\text { area) }\end{array}$ & $\begin{array}{c}\text { Storage } \\
\text { area } \\
\text { (percent } \\
\text { of } \\
\text { subbasin } \\
\text { area) }\end{array}$ & $\begin{array}{l}\text { Main } \\
\text { channel } \\
\text { length } \\
\text { (miles) }\end{array}$ & $\begin{array}{c}\text { Main } \\
\text { channel } \\
\text { slope } \\
\text { (foot } \\
\text { per mile) }\end{array}$ \\
\hline & First Rank Second Rank Third Rank & & & & & & & & & & & & \\
\hline 2804301 & $\begin{array}{l}\text { Unnamed tributary above gaging station near } \\
\text { Mankato: no station number assigned }\end{array}$ & $\mathrm{NW}^{1} /{ }_{4} \mathrm{NW}^{1 / 4}$ & 15 & $108 \mathrm{~N}$ & $27 \mathrm{~W}$ & 1.04 & 0.0 & 0.0 & 1.04 & 0.0 & 0.0 & 2.11 & 129.0 \\
\hline 2804300 & Minnesota River above Blue Earth River & $\mathrm{NW} / 4 \mathrm{NE}^{1 / 4}$ & 14 & $108 \mathrm{~N}$ & $27 \mathrm{~W}$ & 9.73 & .1 & .8 & 11,400 & 2.8 & 5.6 & 326. & 1.4 \\
\hline 3009200 & Blue Earth River above mouth & $\mathrm{NW}^{1} /{ }_{4} \mathrm{NE}^{1 / 4}$ & 14 & $108 \mathrm{~N}$ & $27 \mathrm{~W}$ & 3,540 & 1.6 & 2.7 & 3,540 . & 1.6 & 2.7 & 141. & 2.2 \\
\hline 2804203 & $\begin{array}{l}\text { Minnesota River above gaging station at Mankato: } \\
\text { station number is } 05325000\end{array}$ & $\mathrm{NW}^{1} /{ }_{4} \mathrm{NE}^{1} / 4$ & 13 & $108 \mathrm{~N}$ & $27 \mathrm{~W}$ & 0.97 & .6 & .6 & 14,900 & 2.5 & 4.9 & 327. & 1.4 \\
\hline 2804202 & $\begin{array}{l}\text { Warren Creek above gaging station at Mankato: no } \\
\text { station number assigned }\end{array}$ & $\mathrm{NW}^{1} /{ }_{4} \mathrm{NW}^{1} / 4$ & 18 & $108 \mathrm{~N}$ & $26 \mathrm{~W}$ & 2.69 & .1 & .1 & 2.69 & .1 & .1 & 3.60 & 67.1 \\
\hline 2804201 & $\begin{array}{l}\text { Minnesota River above outfall from sewage treatment } \\
\text { plant for Mankato }\end{array}$ & $\mathrm{SW}^{1 / 4} \mathrm{SW}^{1 / 4}$ & 06 & $108 \mathrm{~N}$ & $26 \mathrm{~W}$ & 11.0 & .2 & .9 & 14,900 . & 2.5 & 4.9 & 328. & 1.3 \\
\hline 2804200 & $\begin{array}{l}\text { Minnesota River above unnamed tributary (subbasin } \\
\text { 2804100) }\end{array}$ & $\mathrm{NE}^{1} / 4 \mathrm{SE}^{1} / 4$ & 25 & $109 \mathrm{~N}$ & $27 \mathrm{~W}$ & 11.8 & .5 & .5 & 14,900 . & 2.5 & 4.9 & 332. & 1.3 \\
\hline 2804100 & Unnamed tributary to Minnesota River above mouth & $\mathrm{NE} / 4 \mathrm{SE}_{4}^{1 / 4}$ & 25 & $109 \mathrm{~N}$ & $27 \mathrm{~W}$ & 4.87 & .1 & 1.0 & 4.87 & .1 & 1.0 & 6.59 & 48.6 \\
\hline 2804001 & $\begin{array}{l}\text { Unnamed tributary above gaging station near } \\
\text { Mankato: station number is } 05325100\end{array}$ & $\mathrm{SW}^{1} / 4 \mathrm{SW}^{1 / 4}$ & 13 & $109 \mathrm{~N}$ & $27 \mathrm{~W}$ & 0.23 & 0 & 0 & .23 & 0 & 0 & 1.02 & 219.1 \\
\hline 2803200 & $\begin{array}{l}\text { Unnamed tributary to unnamed tributary (subbasin } \\
\text { 2803400) above mouth }\end{array}$ & $\mathrm{SW} /{ }_{4} \mathrm{NW} / 1 / 4$ & 20 & $109 \mathrm{~N}$ & $26 \mathrm{~W}$ & 7.89 & 7.3 & 10.2 & 7.89 & 7.3 & 10.2 & 8.49 & 26.2 \\
\hline 2803400 & Unnamed tributary to Minnesota River above mouth & $\mathrm{SW}^{1} /{ }_{4} \mathrm{NW} / 4$ & 18 & $109 \mathrm{~N}$ & $26 \mathrm{~W}$ & 9.72 & .1 & 1.8 & 17.6 & 3.3 & 5.6 & 10.6 & 30.3 \\
\hline 2804000 & Minnesota River above Sevenmile Creek & $\mathrm{SE} / 4 \mathrm{NW}^{1} / 4$ & 12 & $109 \mathrm{~N}$ & $27 \mathrm{~W}$ & 6.35 & .2 & .3 & 15,000 & 2.5 & 4.9 & 338. & 1.3 \\
\hline 2806200 & $\begin{array}{l}\text { County Ditch No. 13A to Sevenmile Creek above } \\
\text { mouth }\end{array}$ & $\mathrm{SE}^{1 / 4} \mathrm{SE}^{1 / 4}$ & 33 & $110 \mathrm{~N}$ & $27 \mathrm{~W}$ & 15.6 & 1.7 & 2.9 & 15.6 & 1.7 & 2.9 & 7.35 & 3.2 \\
\hline 2806600 & $\begin{array}{l}\text { County Ditch No. } 46 \text { A to Sevenmile Creek above } \\
\text { mouth }\end{array}$ & $\mathrm{SE}^{1 / 4} \mathrm{SE}^{1 / 4}$ & 33 & $110 \mathrm{~N}$ & $27 \mathrm{~W}$ & 14.2 & .2 & 8.0 & 14.2 & .2 & 8.0 & 8.05 & 2.9 \\
\hline
\end{tabular}


Table 1. Physical characteristics for the Middle Minnesota - Little Cottonwood River Basin-Continued.

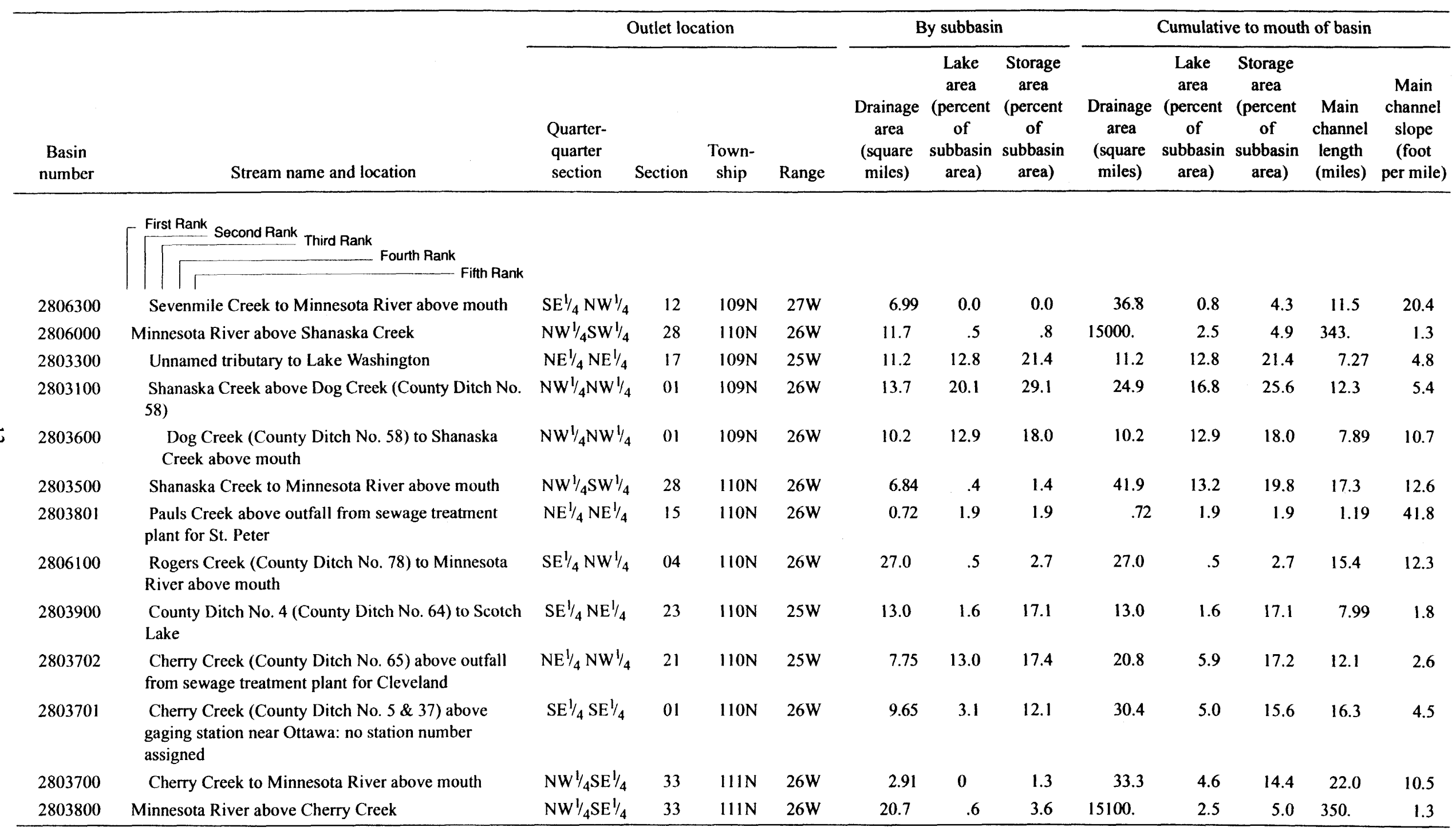

